\title{
ИНТЕНЦИОНАЛЬНОСТЬ СОЗНАНИЯ КАК БИОЛОГИЧЕСКОЕ СВОЙСТВО МОЗГА
}

\begin{abstract}
Аннотация. В статье рассматривается понятие интенциональности в интерпретации Джона Сёрла, которое является краеугольным камнем его философской программы. Основная идея концепции интенциональности Сёрла состоит в том, что интенциональность, так же, как и сознание, должно быть объяснено с естественнонаучной точки зрения, то есть натурализовано. По убеждению философа, если в нашем распоряжении имеется натурализованное понятие интенциональности, то появляется возможность решить множество важных философских проблем. Разум обладает разными формами интенциональности, среди которых основными являются восприятие и интенциональное действие, из которых в свою очередь происходит языковая направленность.

Так как проводимый в рамках исследования анализ имеет проблемную направленность, т.е. главным предметом изучения является интенциональность как свойство мозга, а более конкретно, трактовка данного понятия $c$ точки зрения отдельного представителя англоязычной аналитической философии Дж. Сёрла, то в основном в статье использовался метод теоретической реконструкции, предполагающий анализ лишь тех аспектов философских концепций рассматриваемых теоретиков, что непосредственно связаны с темой статьи.

Подход Сёрла к интенциональности предполагает рассмотрение сознания как естественного биологического феномена. Однако, отличаясь от других феноменов такого рода, сознание является субъективным, и в тоже самое время способным влиять на чисто физиологические процессы. В своей теории интенциональности Сёрл признаёт себя интерналистом, реалистом и последователем Г. Фреге. Он считает субъективный квалитативный аспект более фундаментальным в концепции ментального и употребляет понятие интенциональность для определения подклассов ментальных состояний, которые обладают характеристикой направленности на объект.

Ключевые слова: Сёрл, интенциональность, свойство мозга, интенциональное содержание, ментальные состояния, иллокутивная сила, направление соответствия, условия выполнимости, ментальная репрезентация, возможный мир.
\end{abstract}

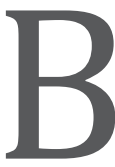

настоящее время объяснение сущности сознания всё ещё напрямую зависит от понимания интенциональности и природы интенционального состояния, а понятие интенциональности представляется одним из основных в современной аналитической философии, которое также применяется в когнитивных науках для обозначения одного из свойств сознания.

В философии Дж. Сёрла понятие интенциональности является ключевым и определяется им, как «свойство ментальных состояний, с помощью которого они могут быть направлены на объекты и положения дел в мире» ${ }^{1}$. Материалистическая

\footnotetext{
1 Searle J. Intentionality: An Essay in the Philosophy of Mind.
} Cambridge, 1983. P. 3. теория сознания Сёрла (часто именуемая как биологическая) подразумевает анализ интенциональности сознания как естественного биологического свойства мозга. Основная идея концепции интенциональности Сёрла состоит в том, что интенциональность, также, как и сознание, должно быть объяснено с естественнонаучной точки зрения, а значит натурализовано.

Центральной задачей философа в данном аспекте являлось примирение понятий сознания и тела: ему необходимо было продемонстрировать, что интенциональность не противоречит его онтологической приверженности научному натурализму. Когда у Сёрла имеется в распоряжении натурализованное понятие интенциональности, то появляется возможность решить множество важных философских 
проблем. С помощью интенциональности Сёрл отделяет действия от поведения, а также использует некоторые свойства интенционального состояния, чтобы объяснить речевые акты, не сводя их просто к действиям или каузальным процессам.

Интенционализм Пола Грайса явился отправным моментом, с которого философы аналитического направления начали рассматривать значение с интенционалистской точки зрения. Брентановский интенционализм сводит ментальные состояния к интенциональным. Сёрл, ключевая фигура данного исследования, скорее присоединяется к интенционализму П. Грайса относительно значения, и не признаёт интенционализм Ф. Брентано.

Пол Грайс был первым, кто связал лингвистическое значение с ментальным состоянием, что способствовало дальнейшему рассмотрению значения с точки зрения аналитической традиции. Интенционализм Грайса очевидно подразумевает интерпретацию лингвистического значения в терминах намерения говорящего. Способность схватывать значение связывается Грайсом с намерением нашего языкового акта. Отправной точкой рассуждений Грайса является представление об утверждении как о действии. Ключевая особенность его теории - идея редукции языкового значения, которая функционирует в широком контексте теории происхождения языка.

По замечанию Д. Деннета и Д. Хоглэна, интенциональность является точкой конвергентции аналитической философии сознания и феноменологии ${ }^{2}$. Феномен интенциональности находится в центре данных двух традиций, при этом феноменология была ассимилирована аналитическими философами, включая Сёрла, что сыграло значительную роль в становлении философии сознания.

Когда Сёрл впервые начал работать над книгой «Интенциональность», он проанализировал всю существующую литературу по этой теме. Материал аналитической философии показался ему довольно непроработанным. Традиционно считается, что лучшее, что написано по поводу природы интенциональности - это обмен мнениями между Р. Чизолмом и У. Селларсом на предмет интенциональности ${ }^{3}$, однако, Сёрл обратил внимание, что в этом диалоге

2 Dennett D.C. Haugeland J. Intentionality // The Oxford Companion to the Mind. 1987. P. 24.

3 Chisholm R., Sellars W. Intentionality and the Mental // Minnesota Studies in the Philosophy of Science. Vol. 2 / ed. Feigl H., Scriven M., and Maxwell G., Minneapolis, 1958. P. 529-539. происходит постоянная путаница между интенциональностью и интенсиональностью. «Многие утверждения об интенциональных состояниях, то есть многие утверждения о желаниях, надеждах и страхах, к примеру, сами являются интенсиональными утверждениями; например, по той простой причине, что они не проходят тесты на замещаемость (закон Лейбница) и экзистенциальное обобщение» 4 . (Это два стандартных теста на интенсиональность). Но тот факт, что утверждения об интенциональных состояниях являются типично интенсиональными утверждениями, не является доказательством того, что существует что-либо по сути интенсиональное в интенциональных состояниях. Использование лингвистических методов часто приводит к путанице свойства описания явления со свойствами самого описываемого явления. Таким образом, Сёрл фактически не почерпнул ничего из переписки ЧизолмаСелларса ${ }^{5}$. Поэтому следующим его шагом было обращение к феноменологам.

Работа «Логические исследования» Эдмунда Гуссерля ${ }^{6}$ откровенно разочаровала Сёрла, так как, ему показалось, что Э. Гуссерль не продвинулся ни на йоту по сравнению с Г. Фреге. Таким образом, Сёрл отказался от попыток выяснить что-то об интенциональности у предшествующих авторов и попросту начал работать над этим вопросом самостоятельно.

Только спустя семь лет напряженного труда выходит работа Сёрла «Интенциональность: Эссе по Философии Сознания» ${ }^{7}$. Когда книга была опубликована, Сёрл столкнулся с тем, что практически каждый её прочитавший, трактовал её как работу гуссерлианского толка, считая, что Сёрл в той или иной степени подражает Гуссерлю, и принимает его подход к интенциональности. Это положение вещей возмутило Сёрла. В качестве ответа на такую неожиданную реакцию философского сообщества выходит его статья «Феноменологическая иллюзия ${ }^{8}$, в которой Сёрл утверждает, что у Гус-

4 Searle J. The Phenomenological Illusion // Experience and Analysis, Proceedings of the 27th International WittgensteinSymposium / ed. Reicher M., and Marek J., 2005. P. 18.

5 Ibid.

6 Husserl E. Logical Investigations. 2 Volumes. London, 1970.

7 Searle J. Intentionality: An Essay in the Philosophy of Mind. Cambridge, 1983.

8 Searle J. The Phenomenological Illusion // Experience and Analysis, Proceedings of the 27th International WittgensteinSymposium / ed. Reicher M., and Marek J., 2005. P. 17-36. 


\section{Материк бессознательного}

серля почерпнуть по данному вопросу ему было просто нечего, и, что на самом деле это были работы Фреге и Виттгенштейна, которые оказали на него существенное влияние 9 .

Ирония также заключалась и в том, что в процессе работы над «Интенциональностью» Сёрл активно дискутировал с экспертами-гуссерлианцами, особенно, с Д. Феллесдалом, который утверждал, что версия интенциональности Гуссерля превосходит концепцию интенциональности Сёрла по целому ряду параметров. Несомненно, между подходом Сёрла к интенциональности и взглядами Гуссерля существуют некоторые совпадения, так как они всё же обсуждают один и тот же предмет.

Хьюберт Дрейфус также активно дискутировал на тему гуссерлианства в подходе Сёрла к интенциональности. Результатом стала довольно длительная дискуссия, описанная в критических публикациях Дрейфуса в адрес сёрловского подхода ${ }^{10}$.

Основное затруднение заключается в том, что феноменологи не понимают вопроса, которым задаётся Сёрл, они усматривают своего рода картезианство в противопоставлении сферы ментального сфере физического (думаю - значит существую) ${ }^{11}$. И на самом деле тот же Х. Дрейфус неоднократно называл Сёрла картезианцем. Такая интерпретация, по мнению Сёрла, в корне неверна: «Ментальная реальность является частью единой реальности ... Вопрос «Каким образом ментальная реальность соотносится с более фундаментальной реальностью?» является не более картезианским, чем вопрос «Как химия соотносится с более фундаментальной атомной физикой?» ${ }^{12}$.

В своей статье Дрейфус пишет: «Нам следует принять онтологию сильнее картезианской онтологии сознания и природы, принятой Хассерлом и Сёрлом» ${ }^{13}$. Он добавляет, что «мы должны следовать Мерло-Понти, приняв за аксиому «третий вид бытия» ${ }^{14}$. Посылка такова, что уже существует два разных вида бытия, сознание и природа, и, что

\section{Ibid.}

10 Dreyfus H. The Primacy of Phenomenology Over Logical Analysis: A Critique of Searle, 1999.

11 Dreyfus H. Hall H. Husserl, Intentionality and Cognitive Science. MIT, 1982.

12 Searle J. The Phenomenological Illusion., 2005. P. 19.

13 Dreyfus H. The Primacy of Phenomenology Over Logical Analysis: A Critique of Searle, 1999. P. 21.

14 Ibid. нам необходимо принять за аксиому «третий вид бытия». Сама терминология «сознание и природа» и «третий вид бытия» делает невозможным обращение ко многим фундаментальным вопросам философии. По мнению Сёрла, сознание не противопоставлено природе, так как сознание является частью природы, и не существует трёх видов (форм) бытия, потому что нет и двух форм бытия, и даже одной его разновидности, так как понятие «бытие» в целом ставит в тупик ${ }^{15}$.

По Сёрлу, невозможно переоценить ведущую роль феноменологии в современной философии сознания. По его мнению, тот факт, что мы признаем, что сознание, со всей его феноменологичностью, является производным и зависящим от базовых фактов реальности свидетельствует о том, что феноменология играет существенную роль в анализе проблем, связанных с сознанием. Однако, феноменологическое исследование - это только начало, затем нужно идти дальше и исследовать логические структуры, большинство из которых редко доступны феноменологии ${ }^{16}$.

Итак, в своей работе «Интенциональность» Сёрл поднимает один из самых фундаментальных вопросов философии языка: каким образом язык описывает факты действительности? А именно, каким образом высказывание обладает значением и направленностью на объект, при том, что оно всего лишь звучит и маркирует, что является лишь его физическим выражением. Сёрл даёт основательный ответ на данный вопрос, который кратко можно свести к следующему тезису: высказывание обладает значением посредством его выведения из внутренней (intrinsic) и базовой интенциональности сознания. Разум обладает разными формами интенциональности, среди которых основными являются восприятие и интенциональное действие, а направленность языка происходит из них ${ }^{17}$.

На самом деле в своей теории интенциональности Сёрл признаёт себя интерналистом, реалистом и последователем Г. Фреге. Интернализм это положение о том, что всё интенциональное содержание находится полностью «в голове» и предполагает возможность непосредственного доступа

15 Searle J. The Phenomenological Illusion // Experience and Analysis, Proceedings of the 27th International WittgensteinSymposium / ed. Reicher M., and Marek J., 2005. P. 20.

16 Ibid.

17 Searle J. Intentionality: An Essay in the Philosophy of Mind. Cambridge, 1983. P. 82. 
в акте рефлексии к состоянию собственного сознания. Интенциональное содержание всего перцепционного опыта, убеждений, желаний, высказываний состоит исключительно из того, что является внутренним для того, кто ими обладает. Интенциональное содержание составлено как из лингвистических, так и нелингвистических репрезентаций. Интенциональное содержание тем не менее не включает объектов и событий реального мира или моментов вне сознания, о которых человек размышляет. Такие объекты, события и моменты могут быть поняты в условиях выполнимости интенционального содержания, но не в самом содержании.

Теория интенциональности Сёрла - «по духу» фрегевская. Сёрл перенял центральный принцип Фреге о том, что любая направленность обеспечена качеством объектов «удовлетворяющих» или подходящих интенциональному содержанию. Наш перцептивный опыт, мысли и высказывания направлены на определённые объекты, потому что эти объекты соответствуют определённым условиям удовлетворённости.

Сёрловская теория интенциональности подразумевает наивный реализм: объекты нашего перцептивного опыта направлены на объекты реального мира. В перцептивном опыте воспринимающий прямо воспринимает интенциональные объекты, которые удовлетворяют интенциональному содержанию его перцептивного опыта. Наивный реализм контрастирует здесь с теориями, согласно которым никто прямо не воспринимает объекты реального мира.

Философ выделяет следующие виды интенциональности: внутренняя интенциональность, которая представляет собой интенциональность в собственном смысле слова, интенциональность «как если бы» и производная интенциональность ${ }^{18}$. В терминологии Сёрла существуют неосознанные интенциональные акты и сознательные неинтенциональные состояния, которые могут пересекаться, но не включают один в другой.

По мнению Сёрла, неправильно было бы утверждать, что каждый раз слушая высказывания, мы придаем определённые значения словам исключительно из-за внутренней интенции говорящего, скорее слушающий апеллирует непосредственно к высказыванию, а не к интенции ${ }^{19}$.

18 Ibid.

19 Ibid. P. 83.
Таким образом, именно языковые высказывания имеют основную значимость, а не субъект, который их воспроизводит. А причиной тому является, по утверждению Сёрла, тот факт, что речь начинает функционировать самостоятельно от субъекта высказывания, создавая эффект своей отдельной интенциональности. То есть, слыша произносимые слова, мы подбираем значение к каждому слову, и это имеет первостепенное значение. В данной кажущейся интенциональности и заключается переход с полностью закрытого для других уровней субъективных состояний сознания на интерсубъективный уровень общения. Так, если бы наша речь не создавала бы такой иллюзии, то не существовало бы тогда способа скоррелировать автономные миры сознаний ${ }^{20}$.

Сёрл проводит параллель между речевыми актами и интенциональными состояниями, описывая интенциональность как репрезентацию, которая аналогична репрезентативной функции речевого акта. Данный параллелизм, по мнению философа, выражается в том, что в интенциональных состояниях, также, как и в речевых актах, существует различие между пропозициональным содержанием и иллокутивной силой, где пропозициональное содержание представляет собой нейтральное высказывание, обладающее неким значением, а иллокутивная сила является неким способом употребления высказывания ${ }^{21}$.

Во-вторых, по версии Сёрла, интенциональным состояниям присуще так называемое «направление соответствия», то есть некоторые речевые акты необходимо соответствуют некому положению дел в мире (фактам), а значит их направление соответствия от «слова к миру». Другие же речевые акты (приказы, императивы) имеют противоположное направление, то есть «от мира к слову», так как их намерение - изменить положение вещей. Точно также, по Сёрлу, различаются и интенциональные состояния ${ }^{22}$.

Следующим связующем звеном речевых актов с интенциональными состояниями Сёрл называет условием выполнимости, то есть каждый речевой акт необходимо подразумевает некое интенцио-

20 McIntyre R. Searle on Intentionality // Inquiry, 27 (1984), 468-483.

${ }^{21}$ Rust J. John Searle (Continuum Contemporary American Thinkers). L.; N.Y., 2009. P. 16.

22 Searle J. Intentionality: An Essay in the Philosophy of Mind. Cambridge, 1983. P. 93. 


\section{Материк бессознательного}

нальное состояние, которое и является условием выполнимости, то есть человек, как правило, верит сам в то что говорит, например: «На улице жарко». Условия соответствия речевого акта и реальности, так же, как и условия соответствия неких убеждений существующим вещам и явлениям Сёрл и называет «условиями выполнимости».

Сёрл делает вывод, что интенциональное состояние с тем или иным направлением соответствия представляет собой репрезентацию своих условий выполнимости.

Таким образом, Сёрл характеризовал типы речевых актов в терминах «условий» или «правил», которым они должны соответствовать, включая условия их пропозиционального содержания. Если приказать Джону вымыть посуду, содержанием императива будет являться пропозиция того, что Джон будет мыть посуду. Точно такая же пропозиция будет содержанием утверждения «Я сказал Джону помыть посуду» или вопроса: «Я спросил, будет ли Джон мыть посуду?». «В то время как вопросы или утверждения могут иметь любую пропозицию своим содержанием, императивы и директивы предполагают некие ограничения: их содержанием может быть только пропозиция, касающаяся будущих действий слушателя, как в данном примере. Подобно тому, как содержание обещаний может являться будущим действием говорящего» 23 .

В более поздних работах Сёрл сделал акцент на схожести речевых актов с ментальными состояниями. И те и другие обладают пропозициональным содержанием и дуальной структурой. При этом ментальные состояния имеют «психологический модус», детерминирующий направление соответствия, а речевые акты обладают иллокутивной силой.

Интенциональное состояние, по Сёрлу, - это визуальный опыт (модус), содержание которого является конъюнктивом, чей второй конъюнкт относится непосредственно к опыту, о котором идёт речь, в то время как первый определяет «воспринимаемое положение дел» ${ }^{24}$. Пропозициональное содержание состояния для него - это то, что определяет его условия выполнимости:

«Каждое интенциональное состояние включает в себя некоторое интенциональное содержание

\footnotetext{
23 Searle, 1969. P. 57-58.

24 Searle J. Intentionality: An Essay in the Philosophy of Mind. Cambridge, 1983. P. 48.
}

в определенном психологическом модусе. Там, где это содержание оказывается полным суждением и где имеется направление соответствия, интенциональное содержание детерминирует условия выполнимости. Условия выполнимости, детерминированные интенциональным содержанием, осуществлены, если состояние выполнено. Благодаря этому спецификация содержания уже является спецификацией условий выполнимости. Таким образом, если я убежден, что идет дождь, то содержанием моего убеждения будет: идет дождь, а условиями выполнимости: идет дождь, а не то, например, что земля мокрая или что с неба льет вода. Поскольку всякая репрезентация - будь то мысль, язык, рисунок или что-либо еще - всегда репрезентация под определенным углом зрения, постольку условия выполнимости репрезентированы под определенным углом зрения» 25 .

В соответствии с данной характеристикой интенционального содержания, вполне оправданно использовать всеобщие условия выполнимости воспринимаемого опыта как основание для определения его содержания. Следовательно, содержание визуального опыта - само - референциально. Однако, отметим, что невозможно одновременно утверждать, что содержание является тем, что определяет условия удовлетворённости и, что содержание не зависит от модуса ${ }^{26}$.

Сёрл разделяет ментальное на интенциональные и неинтенциональные феномены ${ }^{27}$. В то время, как и те и другие потенциально сознательны, интенциональные ментальные состояния направлены на объект, а неинтенциональные состояния нет. Неинтенциональные состояния такие, например, как боль, не могут быть «о чём-либо» и считаются ментальными феноменами, потому что обладают необходимой феноменологией, им нет необходимости отсылать к чему-то вне себя.

Репрезентализм при этом рассматривает характеристику интенционального состояния направленность на как определяющий признак ментального, далее уже разделяя ментальное в соответствии с тем, будет ли оно представлять собой сознательное состояние или нет. То есть все

\footnotetext{
25 Ibid. P. 12-13.

26 John Searle's Philosophy of Language: Force, Meaning and Mind / ed. Tsohatzidis S. L., Cambridge, 2007.

27 Searle J. Intentionality: An Essay in the Philosophy of Mind. Cambridge, 1983.
} 
ментальные состояния, включая боль, - интенциональны. Боль можно трактовать как состояние, отсылающее к чьему-либо телу. Будучи феноменалистом, Сёрл считает субъективный квалитативный аспект более фундаментальным в концепции ментального и хотя он согласен с репрезенталистами в том, что многие ментальные состояния направлены на объект, он употребляет понятие интенциональность для определения подклассов ментальных состояний, которые имеют характеристику направленности на объект ${ }^{28}$.

Рассмотрим теперь, как именно, по мнению Сёрла, интенциональные состояния способны к репрезентации действительности. В рассуждениях Сёрла, аналогия между интенциональными состояниями и моделями, образами и предложениями рушится довольно быстро:

«Когда я говорю, например, что убеждение представляет собой репрезентацию, я вовсе не утверждаю, что убеждение - это некий образ или трактовка значения, а также и не считаю, что убеждение репрезентирует что-то, что было репрезентировано до, и не говорю, что убеждение обладает значением...» ${ }^{29}$

Сёрл пытается объяснить способность моделировать значение или образы посредством обращения к способности сознания к репрезентации. В тоже время в других работах Сёрл делает акцент на аналогии между ментальной репрезентацией и моделированием (или представлением «в образах»). Как ментальная репрезентация, так и моделирование представляют мир в определённом модусе, а именно в соответствии с условиями удовлетворённости.

Как ментальная репрезентация, так и моделирование могут интерпретировать мир в верном и неверном ключе, с разной степенью идеализации. Разница состоит в том, что образы приобретают свою способность к репрезентации не только посредством физического отражения мира, а также и посредством нашей внутренней способности к ментальной репрезентации ${ }^{30}$. Хотя Сёрл и прав, отрицая, что интенциональные состояния подобны

28 Ibid.

29 Ibid. P. 11-12.

30 Searle "Meaning, Communication, and Representation", in Philosophical Grounds of rationality: Intentions, Categories, Ends, ed. Richard E. Grandy and Richard Warner. Oxford: Clarendon Press, 1986. изображениям в сознании ${ }^{31}$, тем не менее данное сравнение помогает в определении трёх особенностей сёрловской интерпретации понятия интенциональности. Во-первых, и самое основное - это аналогия между моделями и интенциональными состояниями иллюстрирует смысл, в котором для Сёрла ментальные состояния являются фундаментально гипотетическими.

Как моделирование, так и репрезентация влечёт за собой человеческую способность увидеть мир или вселенную иначе, чем это могло быть в реальности. Вполне возможно представить мир, в котором, например, нет людей, и в то же самое время, можно представить и наш обычный мир. Модель вселенной - это не сама вселенная, это своего рода описание или отражение образа вселенной ${ }^{32}$. Так как интенциональное состояние отражает объект и одновременно отличимо от него, так же может быть и наоборот. Ментальные репрезентации и модели представляют возможную вселенную, которая находится в различных отношениях с действительной вселенной: эти отношения позволяют говорить, как об интенциональных состояниях, так и о моделях в терминах точности, соответствия, несоответствия, заблуждения, неопределённости и др.

Таким образом, как модели, так и ментальные репрезентации отражают возможный мир ${ }^{33}$. Более того, возможная вселенная (BВ) может быть либо идентичной с реальной вселенной (РВ), либо расходится с ней. ВВ и РВ не будут являться изоморфными. Например, я могу представить, что у меня в холодильнике много экзотических фруктов, а на самом деле там их нет. При этом $\mathrm{BB} \neq \mathrm{PB}$.

Представленное содержание нацелено на состояния удовлетворенности интенционального состояния. К сожалению, Сёрл использует фразу «состояние удовлетворенности» в двух разных, но частично совпадающих смыслах. Прежде чем, понять смысл «условия удовлетворенности» нужно сначала понять значение выражения «психологический модус». Это вторая особенность аналогии между интенциональными состояниями и моделями. В той мере как представленный возможный

\footnotetext{
31 Rust J. John Searle (Continuum Contemporary American Thinkers). L.; N.Y., 2009. P. 34.

32 Ibid. P. 35.

33 See Stalnaker R. Assertion in Context and Content: Essays on Intentionality in Speech and Thought. Oxford; New York, 1999. P. 79.
} 


\section{Материк бессознательного}

мир не идентичен реальному миру, ВВ может быть отличима от РВ в двух направлениях: он (мир) может быть точен, но неполон или он может быть просто неточен.

Второй смысл, в котором содержание интенционального состояния может быть не о нашей реальной вселенной, а о некой воображаемой, тогда, когда оно просто отражает неверное положение вещей. Модель или содержание могут быть частично неверными, если они неправильно интерпретирует особенности, которые пытаются репрезентировать.

Существует третий смысл, в котором интенциональное состояние можно сравнить с моделями. Если модель репрезентирует объект, то это необязательно происходит посредством каузальной связи с данным объектом.

Сёрл следующим образом выстраивает свою теорию интенциональности. Интенциональные состояния репрезентируют объекты. Часть интенционального состояния, которая репрезентирует эти объекты, называется репрезентативным содержанием. Репрезентативное содержание аналогично модели. Содержание репрезентирует возможный мир - его условия удовлетворения, которые могут соответствовать или не соответствовать реальному миру.

«Психологический модус» интенционального состояния определяет способ, с помощью которого используется репрезентативное содержание. Можно что-то утверждать, выражать желание, обещать. Например, можно сказать: «Я хочу поехать на Майами» или «Я обещаю поехать на Майами», или «Я съездил на Майами». Таким образом, все эти предложения обладают одним и тем же содержанием «поездка на Майами», но с различным психологическим модусом. Сёрл утверждает, что у нас могут быть различные интенциональные состояния у которых может быть одинаковое репрезентативное содержание в множестве различных вариантов ${ }^{34}$.

C помощью понятия «направление соответствия» Сёрл подразделяет разные психологические модусы на управляемые виды классов. Психологические модусы либо представляют направление соответствия «слова к миру», либо «от мира к слову».

Когда репрезентативное содержание представляет возможный мир, который отличается от

34 Searle J. Meaning, Communication, and Representation // Philosophical Grounds of rationality: Intentions, Categories, Ends / ed. Grandy R., Warner R. Oxford, 1986. P. 144. реального мира (так что содержание частично неверно), различие говорит нам только о том, что существует пробел, который необходимо заполнить. Направление соответствия говорит нам о том, каким образом данный пробел может быть заполнен. Если моё интенциональное содержание репрезентирует возможный мир, где в моём холодильнике множество экзотических фруктов, а на самом деле их там нет, то пробел между условиями удовлетворённости и реальным положением дел (в моём холодильнике) может быть заполнен либо изменением репрезентативного содержания, а значит и изменением условий удовлетворённости или же заполнением моего холодильника фруктами, чтобы положение дел соответствовало репрезентативному содержанию.

Если существует несоответствие между репрезентируемым возможным миром и реальным положением дел, могу ли я заполнить этот пробел посредством изменения интенционального содержания? Я меняю интенциональное содержание, когда моя цель точно отразить реальное положение дел в мире, так что содержание функционирует в поддержку определённого убеждения. У меня ложное убеждение о реальном положении дел в моём холодильнике (оно репрезентирует возможную реальность, в которой в моём холодильнике множество фруктов), я меняю моё убеждение в соответствии с реальным положением дел. Убеждения (beliefs), по Сёрлу, обладают направленностью oт сознания к миру, то есть убеждения должны соответствовать реальному положению дел ${ }^{35}$.

Когда убеждение представляет собой случай некоего психологического модуса, который имеет направление от сознания к миру, то имеются и другие модусы, которые также подпадают под ту же категорию. Например, я могу быть убеждённым, могу предполагать, подозревать, заключать, беспокоиться, бояться, что некоторое содержание может совпадать или не совпадать с реальным положением дел.

Если то же самое репрезентативное содержание представлено в ином психологическом модусе (чем те, которые обладают направлением от-сознания-к-миру как, например, убеждение), то пробел может быть заполнен с помощью изменения реального положения дел. Если содержание функционирует в соответствии с психологическим

35 Rust J. John Searle (Continuum Contemporary American Thinkers). L.; N.Y., 2009. P. 34. 
модусом, то желание иметь в холодильнике экзотические фрукты может быть осуществлено, если я реально заполню мой холодильник фруктами. Желания обладают направлением соответствия от-мира-к-сознанию. Мы можем желать, надеяться, намереваться, собираться изменить реальное положение дел тем или иным способом.

Направление соответствия того или иного интенционального состояния затем имеет способ с помощью которого интенциональные состояния по сути нормативны: интенциональная мысль подразумевает в ее основе возможность быть неверной, или неправильно интерпретировать реальное положение дел. Как ложное убеждение, так и желание предполагает, что мы можем представить мир гипотетически, не таким, каков он есть на самом деле. В случае несоответствия мы вынуждены исправлять данное расхождение с реальным положением дел. Сёрл пишет, что «Интуитивно мы можем сказать, что идея направления соответствия - это ответственность за соответствие» ${ }^{36}$. Далее Сёрл разъясняет более подробно:

«Существо, которое обладает интенциональным состоянием с направлением соответствия, должно быть способно отличить соответствие от несоответствия данному состоянию. Это следует из того факта, что интенциональное состояние это репрезентация его условий выполнимости. Это не означает, что они всегда или почти всегда интерпретируются верно, и не могут восприниматься некорректно, скорее это значит, что у нас есть способность определить их соответствие реальному положению дел» ${ }^{37}$.

Репрезентативное содержание создаёт условия для возможности образования некоего несоответствия между сознанием и миром. Но психологический модус указывает, как нам нужно исправить данное несоответствие - посредством изменения самого репрезентативного содержания (в случае с убеждением) или с помощью изменения реального положения дел (в случае с желанием).

Ограничивая свое внимание убеждениями, Сёрл принимает попытку устранить неясность следующим образом:

«Выражение 'условия выполнимости' несет в себе обычную двусмысленность процесса - продукта и может быть, как требование так и требу-

36 Searle J. Intentionality: An Essay in the Philosophy of Mind. Cambridge, 1983. P. 7.

37 Ibid. P. 177. емое. Так, например, если я убежден в том, что идет дождь, то условием выполнимости моего убеждения является то, что должен идти дождь (требование). Именно этого требует мое убеждение для того, чтобы быть истинным. Если же мое убеждение на самом деле истинно, то в мире будет существовать определенное условие, а именно что идет дождь (требуемое), являющееся условием выполнимости моего убеждения, т.е. условие, которое реально выполняет мое убеждение. Я полагаю, что эта двусмысленность совершенно безопасна и даже полезна, если осознавать ее с самого начала» ${ }^{38}$.

Отметим, что интенциональные состояния, по Сёрлу, обладают двумя компонентами. Во-первых, у них есть содержание, которое репрезентирует возможное положение дел или условия выполнимости. Именно это репрезентативное содержание делает возможным интенциональное состояние быть направленным на. Во-вторых, это содержание может быть представлено в том или ином психологическом модусе, который и определяет, где расположено несоответствие между условиями выполнимости и реальным положением дел: если психологический модус - утверждение, значит проблема с содержанием, если - желание, то необходимо менять положение дел в мире, чтобы привести его в соответствие с содержанием. Сёрл объединяет данные положения одной формулой: $S(r)$, где $S$ - психологический модус, a $r$ - peпрезентативное содержание.

Итак, Сёрл придал интенциональным состояниям «аспектуальный» характер, иными словами, они репрезентируют предмет или явление в определенном аспекте или соотносятся с той или иной категорией. Помимо этого, по мнению Сёрла, всякое интенциональное состояние, имеет интенциональное содержание и психологический модус. Базовыми психологическими модусами Сёрл считает восприятие и намерение, однако в классификации в роли основных модусов он рекомендует использовать их производные формы - убеждение (belief) и желание (desire). Остальные интенциональные состояния содержат некие убеждения и желания, и во многих случаях интенциональность таких состояний может быть объяснена какимилибо убеждениями и желаниями.

Но основной трудностью Сёрл считает доступное объяснение отношения ментальных состояний к элементарным частицам. Тем не менее, по

38 Ibid. P. 13. 
его мнению, сознательные ментальные состояния каузально порождены мозгом и в нём же реализуются. Данный подход предполагает рассмотрение сознания как естественного биологического феномена. Однако, по мнению Сёрла, отличаясь от других феноменов такого рода, сознание является субъективным, и в тоже самое время способным влиять на чисто физиологические процессы, например, в случае, когда осознанное желание совер- шить какое-либо действие вызывает определенное поведение.

С целью иллюстрации такой «интенциональной каузальности», в своих дальнейших работах по философии сознания Сёрл провёл параллель между сознанием, которое порождается работой нейронов, и явлениями природы, когда свойства целого невозможно свести к свойствам составляющих их частей.

\section{Список литературы:}

1. Chisholm R., Sellars W. Intentionality and the Mental // Minnesota Studies in the Philosophy of Science. Vol. 2 / ed. Feigl H., Scriven M., and Maxwell G., Minneapolis, 1958, pp. 529-539.

2. Dennett D.C. Haugeland J. Intentionality. // The Oxford Companion to the Mind. 1987, pp. 160-64.

3. Dreyfus H., Hall H. Husserl Intentionality and Cognitive Science. MIT, 1982.

4. $\quad$ Dreyfus H. The Primacy of Phenomenology Over Logical Analysis: A Critique of Searle, 1999.

5. Husserl E. Logical Investigations. 2 Volumes. London, 1970.

6. John Searle's Philosophy of Language: Force, Meaning and Mind / ed. Tsohatzidis S. L., Cambridge, 2007.

7. McIntyre R. Searle on Intentionality // Inquiry, 27. 1984, pp. 468-483.

8. $\quad$ Rust J. John Searle (Continuum Contemporary American Thinkers). L.; N.Y., 2009.

9. Searle J. Intentionality: An Essay in the Philosophy of Mind. Cambridge, 1983.

10. Searle J. Meaning, Communication, and Representation // Philosophical Grounds of rationality: Intentions, Categories, Ends / ed. Grandy R., Warner R., Oxford, 1986, pp. 143-158.

11. Searle J. The Phenomenological Illusion // Experience and Analysis, Proceedings of the 27th International Wittgenstein-Symposium / ed. Reicher M., and Marek J., 2005, pp. 17-36.

12. Stalnaker R. Assertion in Context and Content: Essays on Intentionality in Speech and Thought. Oxford; New York, 1999.

\section{References (transliteration):}

1. Chisholm R., Sellars W. Intentionality and the Mental // Minnesota Studies in the Philosophy of Science. Vol. 2 / ed. Feigl H., Scriven M., and Maxwell G., Minneapolis, 1958, pp. 529-539.

2. Dennett D.C. Haugeland J. Intentionality. // The Oxford Companion to the Mind. 1987, pp. 160-64.

3. Dreyfus H., Hall H. Husserl Intentionality and Cognitive Science. MIT, 1982.

4. Dreyfus H. The Primacy of Phenomenology Over Logical Analysis: A Critique of Searle, 1999.

5. Husserl E. Logical Investigations. 2 Volumes. London, 1970.

6. John Searle's Philosophy of Language: Force, Meaning and Mind / ed. Tsohatzidis S. L., Cambridge, 2007.

7. McIntyre R. Searle on Intentionality // Inquiry, 27. 1984, pp. 468-483.

8. Rust J. John Searle (Continuum Contemporary American Thinkers). L.; N.Y., 2009.

9. Searle J. Intentionality: An Essay in the Philosophy of Mind. Cambridge, 1983.

10. Searle J. Meaning, Communication, and Representation // Philosophical Grounds of rationality: Intentions, Categories, Ends / ed. Grandy R., Warner R., Oxford, 1986, pp. 143-158.

11. Searle J. The Phenomenological Illusion // Experience and Analysis, Proceedings of the 27th International Wittgenstein-Symposium / ed. Reicher M., and Marek J., 2005, pp. 17-36.

12. Stalnaker R. Assertion in Context and Content: Essays on Intentionality in Speech and Thought. Oxford; New York, 1999. 